\title{
Réveil peropératoire et memorisation: prévalence et aspects cliniques dans un Pays Sub-Saharien
}

\section{Martin Lankoandé}

Médecin anesthesiste / Unité de Formation et de Recherche en Sciences de la Santé (UFR/SDS) / Unisversité Ouaga-I Pr Joseph-Ki Zerbo/ Burkina Faso

\section{Papougnezambo Bonkoungou}

Maître Assistant en Anesthésie / UFR SDS /

Unisversité Ouaga-I Pr Joseph-Ki Zerbo/ Burkina Faso

\section{Sylvain Konombo}

Médecin Biologiste / Hôpital Yalgado /

Unisversité Ouaga-I Pr Joseph-Ki Zerbo/ Burkina Faso

Armel Flavien R Kaboré

Professeur en Anesthésie / UFR SDS /

Unisversité Ouaga-I Pr Joseph-Ki Zerbo/ Burkina Faso

\section{Christian Napon}

Professeur enNeurologie / UFR SDS /

Unisversité Ouaga-I Pr Joseph-Ki Zerbo/ Burkina Faso

\section{Joachim Sanou}

Professeur en Anesthésie / UFR SDS /

Unisversité Ouaga-I Pr Joseph-Ki Zerbo/ Burkina Faso

\section{Bernard Le Polain De waroux}

Professeur Anesthésie / Université Catholique de Louvain /Belgique

\section{Nazinigouba Ouédraogo}

Professeur Anesthésie Réanimation / UFR SDS /

Unisversité Ouaga-I Pr Joseph-Ki Zerbo/ Burkina Faso

Abstract

Objective: To study intraoperative memorization in the service of general surgery of Yalgado Ouedroago teaching Hospital (YO-TH) in Burkina Faso.

Methods: It was a prospective study in the service of general surgery of YOTH over 7 months. The inclusion criteria were: age $\geq 18$ years, absence of psychiatric disorder, American Society of Anesthesiology (ASA) score less than 4 , informed consent written. Patients were interviewed at recovery. The 
questionnaire of Brice et al [8] was used and data were analyzed with SPHINX version 5.0.1

Results: A total of 475 patients were included. The mean age was $40.4 \pm$ 16.3 years. The sex ratio was 1.4 . The majority (56\%) took psychoactive substance and $78.1 \%$ of patients had ASA score 2 or 3. Seven $(1.4 \%)$ patients reported an explicit perioperative memorization $(\mathrm{EPOM}+)$. The mean age of EPOM + patients was $26.8 \pm 6.57$ years with a sex ratio 1.3. There was difference between EPOM + patients and EMPO - for age ( $\mathrm{p}=$ $0.0001)$, occupation $(\mathrm{p}=0.009)$ and antecedent of local anesthesia $(\mathrm{p}=$ 0.004). Five patients reported auditory perceptions, four cases of visual perceptions and two tactile perceptions. Pain was the most unpleasant memorization recorded. The psychological consequences of EPOM + were anxiety ( 2 cases).

Conclusion: The frequency of EPOM is low in our context and the main cause was a slight anesthesia.

Keywords: Memorization, Surgery, General Anesthesia

\section{Résumé}

Objectif : Etudier la mémorisation peropératoire en chirurgie générale à 1'Hôpital Universitaire Yalgado Ouédroago (HU-YO) au Burkina Faso. Méthodes : Il s'est agi d'une étude prospective menée en chirurgie générale à 1'HU-YO sur 7 mois. Les critères d'inclusion étaient: un âge $\geq 18$ ans, absence de trouble psychiatrique, un score ASA inférieur ou égal à 4, consentement éclairé marqué. Les patients étaient interviewés à leur réveil. Le questionnaire de Brice et al [8] a été utilisé et les données ont été analysées à 1'aide SPHINX version 5.0.1.

Résultats : Au total 475 patients ont été retenus. L'âge moyen était 40,4 \pm 16,3 ans avec un sex ratio de 1,4. La majorité (56\%) prenait des substances psychoactives et avaient un score ASA 2 ou $3(78,1 \%)$. Sept patients $(1,47 \%)$ ont rapporté un épisode de mémorisation per opératoire explicite (MPOE+). L'âge moyen des patients MPOE+ était 26,8 $\pm 6,5$ ans pour un sex ratio de 1,3. Il y avait une différence entre les patients MPOE + et MPOE - pour l'âge $(p=0,0001)$, la profession $(p=0,009)$ et l'antécédent d'anesthésie locorégionale $(p=0,004)$. Cinq patients ont rapporté des perceptions auditives, quatre des perceptions visuelles et deux des perceptions tactiles. La douleur était la mémorisation la plus désagréable. Les conséquences psychologiques de la MPEO+ étaient l'anxiété ( 2 cas).

Conclusion : La fréquence de la MPOE est faible dans notre contexte. La principale cause est une anesthésie légère.

Mots-clés : Mémorisation, Chirurgie, Anesthésie Générale 


\section{Introduction}

Les progrès pharmacologique et technique permettent de réaliser une anesthésie sûre et confortable. En anesthésie générale (AG), la peur de ne pas se réveiller a progressivement été supplantée par celle de ne pas dormir (Myles 2000). Les patients s'attendent à être endormis sans garder de souvenir peropératoire (Snow 1849). Il arrive que ces attentes ne soient pas satisfaites pour des raisons diverses. Ainsi, des réveils et des mémorisations peropératoire (MPO), ou souvenirs d'événements per-opératoires peuvent survenir. On parlera de MPO explicite (MPOE) lorsque le souvenir du réveil peropératoire est conscient et de MPO implicite (MPOI) lorsque le souvenir n'est pas conscient. Ces phénomènes surviennent à des moments où l'anesthésie est peu profonde avec une fréquence de l'ordre de 1\% (Errando 2008). La MPO constitue la première cause d'insatisfaction des patients anesthésiés et la deuxième préoccupation des anesthésistes après le risque de décès (Macario 1999). En Afrique sub-saharienne, des études ont rapporté des fréquences de 1,7\% (Ouro-Bang'na maman 2008). Au Burkina Faso, des cas isolés ont été rapportés. Ce constat a motivé cette étude en vue de faire connaitre davantage ce phénomène.

Matériel et méthodes : Il s'est agi d'une étude transversale avec collecte prospective des données dans le service de chirurgie générale et digestive de 1'HU-YO sur une période 7 mois. Les critères d'inclusion étaient : être opéré sous $\mathrm{AG}$, âge $\geq 18$ ans, score $\mathrm{ASA} \leq 4$, obtention d'un consentement éclairé. Les patients étaient interviewés après leur réveil. La taille de l'échantillon a été estimée à 475 patients pour un intervalle de confiance (IC) de $95 \%$, une proportion estimée entre $0,11 \%$ et $1,89 \%$. Les patients opérés plus d'une fois ont été inclus pour l'opération en cours. Les patients étaient interviewés une seule fois en postopératoire entre $\mathrm{J} 0$ et J14. Les données démographiques, cliniques, anesthésiques et chirurgicales ont été analysées. Aucun moniteur de la profondeur de l'anesthésie n'a été utilisé et les anesthésistes en charge des patients n'ont pas été informés de l'étude. Il y avait MPOE lorsque le patient se rappelait s'être éveillé au moment où il n'était pas supposé 1'être.. Le questionnaire structuré de Brice et al (Brice 1970) a été utilisé. Les données ont été analysées à l'aide du logiciel SPHINX version 5.0.1. Le test de Chi square et de Student ont été utilisés avec une marge d'erreur de 5\%. Nous avons obtenu l'approbation du comité d'Ethique avant la réalisation de l'étude.

\section{Résultats}

Au total 475 patients ont été inclus. L'âge moyen était de 40,4 $\pm 16,3$ ans avec un sex ratio 1,4 . La majorité $(56 \% ; n=266)$ prenait des substances psychoactives. Les scores ASA 3 ou 4 (78,1\%) étaient observés dans 371 
cas. La chirurgie était urgente dans 376 cas $(79,2 \%)$. L'induction anesthésique intraveineuse (IV) associée aux halogénés (Autres Types Anesthésies) était pratiquée 275 cas contre 176 cas d'anesthésie balancée $(\mathrm{AB})$ à préciser. La figure 1 montre la répartition des patients selon la technique d'anesthésie. Vingt-quatre patients ont bénéficié d'une anesthésie intraveineuse pure (ATIV).

\section{Type d'anesthesie}

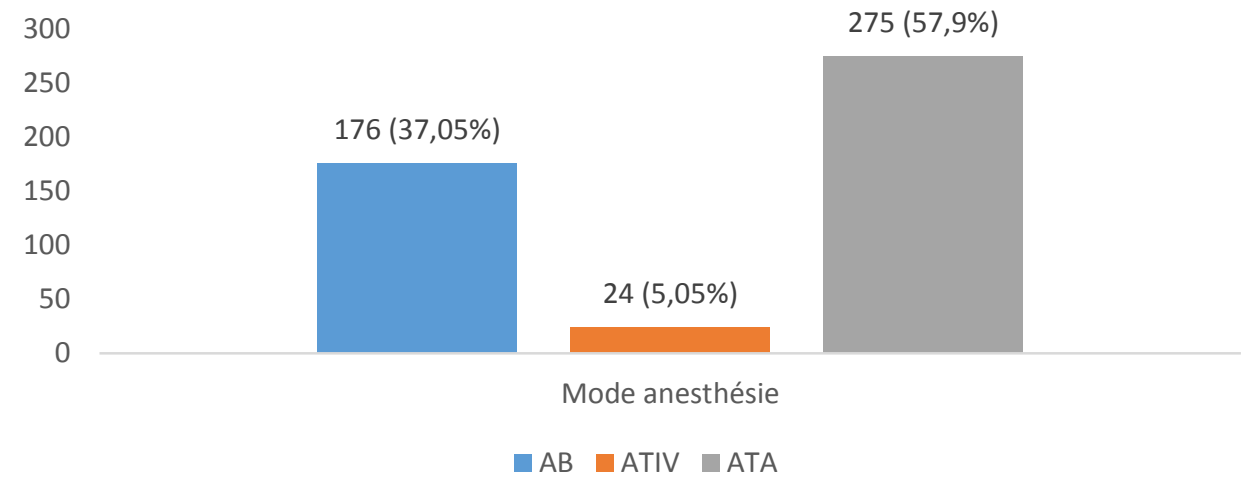

Figure 1 : Répartition des patients selon l'anesthésie.

Sur 475 patients, 7 se rappelaient d'un réveil au cours de leur intervention (MPOE+) dont 4 étaient certains (groupe 1) et 3 douteux (groupe 2). Le tableau I ci-dessous décrit les cas de mémorisation.

Tableau I : Répartition selon la presence de mémorisation $(\mathrm{n}=475)$

\begin{tabular}{ccc}
\hline Interrogatoire & Nombre & Pourcentage \\
\hline MPOE + & & \\
Groupe 1 & 4 & 0,84 \\
Groupe 2 & 3 & 0,63 \\
MPOE - & 468 & 98,63 \\
Total & 475 & 100 \\
\hline
\end{tabular}

L'incidence de MPOE était 1,4\% ( 7 / 475). L'âge moyen des patients MPOE+ était de 26,8 $\pm 6,5$ ans dont 4 hommes et 3 femmes. Parmis les 7 cas de MPOE, des antécedents à type d'ALR (1 cas), d'AG (1 cas), d'ALR + AG (1 cas) étaient observés. Six patients consommaient des substances psychotropes. Les scores ASA 3 ou 4 prédominaient (5/7) comme l'illustre le tableau II.

Tableau II: Données démographiques des patients $(\mathrm{n}=475)$.

\begin{tabular}{cccc}
\hline Variables & MPOE $+\mathbf{N}=\mathbf{7}$ & MPOE- $\mathbf{N}=\mathbf{4 6 8}$ & p \\
\hline \multirow{2}{*}{ Age (années) } & $\mathrm{N}=7$ & $\mathrm{~N}=468$ & \\
\cline { 2 - 3 } & $26,8 \pm 6,5$ & 0,0001
\end{tabular}




\begin{tabular}{|c|c|c|c|}
\hline M & $4 / 7$ & $280(59,8 \%)$ & \\
\hline $\mathrm{F}$ & $3 / 7$ & $188(40,2 \%)$ & \\
\hline ssion & & & 0,0097 \\
\hline ltivateur & & $146(31,3 \%)$ & \\
\hline lénagère & $1 / 7$ & $116(24,8 \%)$ & \\
\hline /SE & $3 / 7$ & $111(23,7 \%)$ & \\
\hline larié & 0 & $55(11,7 \%)$ & \\
\hline Etudiant & $3 / 7$ & $40(8,5 \%)$ & 0,012 \\
\hline \multicolumn{3}{|c|}{ Provenance } & \\
\hline adougou & $5 / 7$ & $284(60,6 \%)$ & \\
\hline Iagadougou & $2 / 7$ & $184(39,4 \%)$ & \\
\hline
\end{tabular}

Il y avait une différence significative entre les patients MPOE+ et MPOEpour l'âge $(\mathrm{p}=0,0001)$ et la profession $(\mathrm{p}=0,009)$. Les patients étaient comparables pour le sexe et la provenance. Le tableau III ci-dessous compare les antécédents, les caractéristiques cliniques des patients.

Tableau III: Caractéristiques cliniques $(n=475)$

\begin{tabular}{|c|c|c|c|}
\hline Variables & MPOE+ N=7 & MPOE- N= 468 & $\mathbf{p}$ \\
\hline & $\mathrm{n}(\%)$ & $\mathrm{n}(\%)$ & \\
\hline Antécédents & $4(57,1)$ & $75(16)$ & \\
\hline $\mathrm{AG}$ & $2 \quad(28,5)$ & $53 \quad(11,3)$ & \\
\hline ALR & $2 \quad(28,5)$ & $22 \quad(4,7)$ & 0,004 \\
\hline Psychotropes & $6 \quad(85,7)$ & $260(55,5)$ & \\
\hline Alcool & $4 \quad(57,1)$ & $168(35,9)$ & \\
\hline Tabac & $2 \quad(28,5)$ & $106(22,6)$ & \\
\hline Thé & $2 \quad(28,5)$ & $84 \quad(17,9)$ & \\
\hline Café & $1 \quad(14,2)$ & $82 \quad(17,5)$ & \\
\hline Cola & $1 \quad(14,2)$ & $63 \quad(13,4)$ & \\
\hline autres* & - & $28 \quad(5,9)$ & \\
\hline Aucune & $1 \quad(14,2)$ & $208(44,4)$ & \\
\hline \multicolumn{4}{|l|}{ Type chirurgie } \\
\hline Digestive & $5 \quad(71,4)$ & $352(75,2)$ & \\
\hline Urologie & $1 \quad(14,3)$ & $28 \quad(5,9)$ & \\
\hline Autres $* *$ & $1 \quad(14,3)$ & $60 \quad(13)$ & \\
\hline Gynécologie & - & $28 \quad(5,9)$ & \\
\hline \multicolumn{4}{|l|}{ Score ASA } \\
\hline 1 ou 2 & $2(28,5)$ & $102(21,8)$ & \\
\hline 3 ou 4 & $5 \quad(71,5)$ & $366(78,2)$ & \\
\hline IMC Moyen & $22,25 \pm 1,7$ & $21,87 \pm 2,8$ & \\
\hline
\end{tabular}

$*$ drogues, $* *$ chirurgie de goitre (1cas) 
Les deux groupes étaient différents $(p=0,004)$ pour l'antécedent d'ALR mais comparables pour le poids, la taille, l'IMC, la consommation de substances psychotropes, les antécédents d'AG, le type de chirurgie et le score ASA. Sur les sept cas de MPOE +, cinq ont rapporté des perceptions auditives, quatre des perceptions visuelles, et deux cas des perceptions tactiles. Un patient a rapporté une douleur (lorsqu'on lui « cousait » la peau). La douleur était la mémorisation la plus désagréable. Les perceptions visuelles, auditives et tactiles étaient perçues comme indifférentes. Ces perceptions étaient survenues en fin d'intervention et ont duré quelques secondes. Les données anesthésiques comparatives des patients sont décrites dans le tableau IV.

Tableau IV : Comparaison des données anesthésiques $(\mathrm{n}=475)$

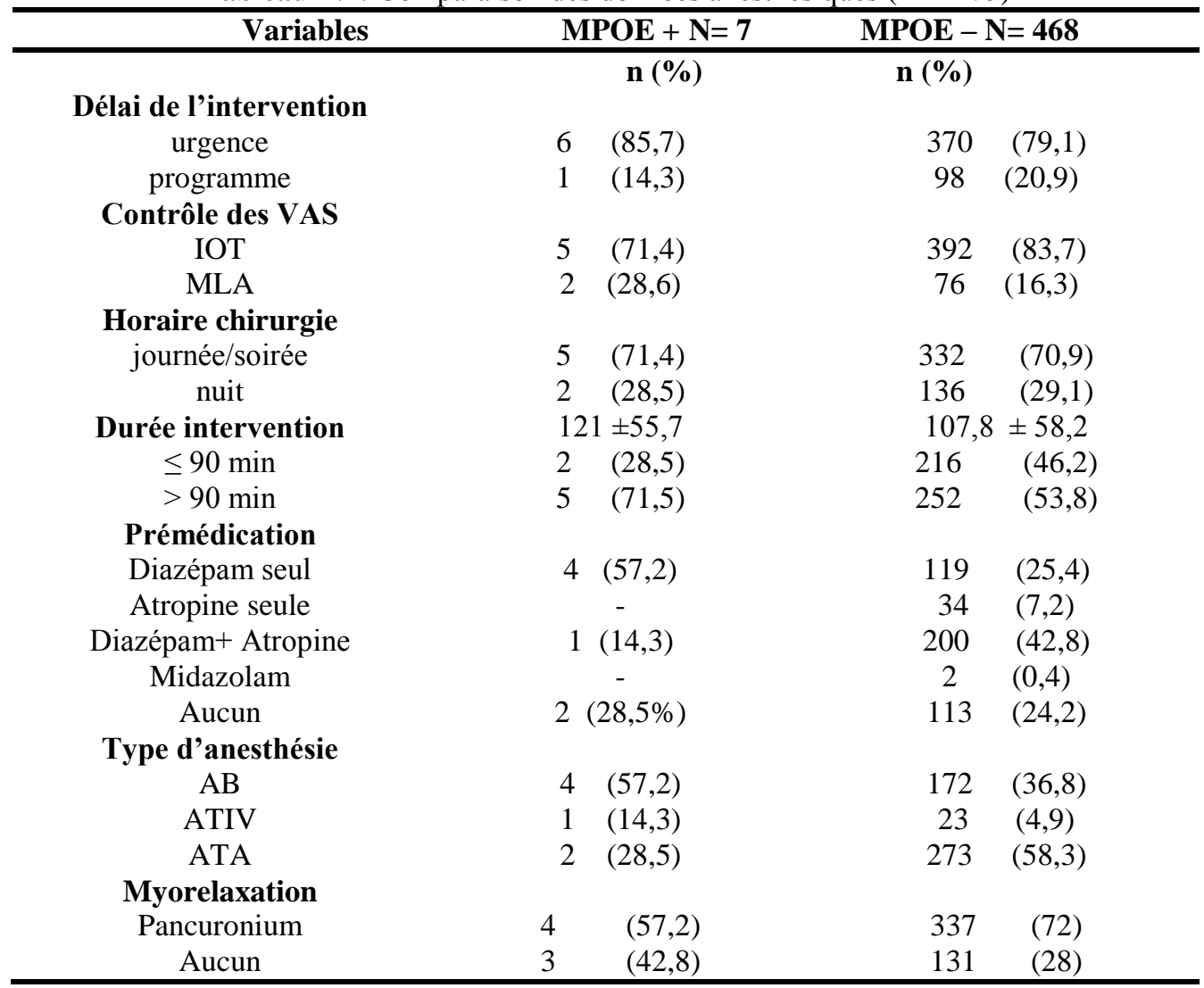

IOT: Intubation Orotrachéale; MLA: Masque Laryngé; AB: Anetshesie Balancée, ATIV: Anesthesie Totale Intraveineuse, ATA: Autre Type Anesthesie

La différence n'était pas significative pour le délai de l'intervention, le mode contrôle des VAS, la prémédication, la durée de l'intervention, l'horaire de l'intervention, le type d'anesthésie, la curarisation. 
Tableau V: Comparaison des doses de médicaments administrés $(\mathrm{n}=475)$

\begin{tabular}{|c|c|c|c|c|}
\hline & \multicolumn{2}{|c|}{ MPOE+ } & & MPOE \\
\hline \multirow{2}{*}{$\begin{array}{c}\text { Agents } \\
\text { Anesthésiques }\end{array}$} & \multicolumn{2}{|c|}{ Dose } & \multicolumn{2}{|r|}{ Dose } \\
\hline & Moyenne 1 & Min-Max & Moyenne & Min-Max \\
\hline \multicolumn{5}{|c|}{ Prémédication } \\
\hline Diazépam ( $\mu \mathrm{g} / \mathrm{kg})$ & $55,3 \pm 21,1$ & $125-181,8$ & $158,2 \pm 22,1$ & $68,5-222,2$ \\
\hline Atropine $(\mu \mathrm{g} / \mathrm{kg})$ & 8,3 & 8,3 & $8 \pm 0,9$ & $4,1-11,1$ \\
\hline Midazolam $(\mu \mathrm{g} / \mathrm{kg})$ & - & - & $88,5 \pm 3,3$ & $86,2-90,9$ \\
\hline \multicolumn{5}{|c|}{ Induction } \\
\hline Propofol (mg/kg) & $3,3 \pm 0,5$ & $2,7-4$ & $3,2 \pm 0,8$ & $0,7-6,5$ \\
\hline Kétamine (mg/kg) & $3 \pm 0,8$ & $2,2-3,8$ & $3,6 \pm 1$ & $1,4-6$ \\
\hline Thiopental (mg/kg) & - & - & $6,6 \pm 2,7$ & $2,9-11,7$ \\
\hline Hypnomidate $(\mathrm{mg} / \mathrm{kg})$ & $\mathrm{kg})$ & - & 0,25 & 0,2 \\
\hline \multicolumn{5}{|l|}{ Entretien } \\
\hline Halothane (\%vol.) & $1,8 \pm 0,3$ & $1,5-2$ & $1,8 \pm 0,4$ & $0,7-3,5$ \\
\hline Isoflurane (\%vol.) & 1,5 & 1,5 & $1,6 \pm 0,2$ & $1,5-2$ \\
\hline Propofol (mg/kg) & $1 \pm 0,3$ & $0,8-1,2$ & $1,5 \pm 1,2$ & $0,5-8$ \\
\hline Kétamine (mg/kg) & 3,1 & 3,1 & $2,9 \pm 1,2$ & $0,4-7,5$ \\
\hline Thiopental (mg/kg) & - & - & $3,2 \pm 0,8$ & $1,9-4,7$ \\
\hline \multicolumn{5}{|c|}{ Analgésie } \\
\hline Fentanyl $(\mu \mathrm{g} / \mathrm{kg})$ & $2,8 \pm 0,8$ & $1,5-3,8$ & $3,3 \pm 0,9$ & $1,4-7,3$ \\
\hline Sufentanil $(\mu \mathrm{g} / \mathrm{kg})$ & - & - & $0,4 \pm 0,2$ & $0,2-0,8$ \\
\hline \multicolumn{5}{|c|}{ Myorelaxation } \\
\hline Pancuronium $(\mu \mathrm{g} / \mathrm{kg})$ & $60,6 \pm 7,4$ & $50-66,6$ & $64,2 \pm 12,3$ & $30,7-142,8$ \\
\hline Atracurium $(\mu \mathrm{g} / \mathrm{kg} /)$ & - & - & $105 \pm 24,4$ & $76,9-120,7$ \\
\hline Succinylcholine $(\mathrm{mg} / \mathrm{kg})$ & g) & - & $1,4 \pm 0,2$ & $1,2-1,5$ \\
\hline Verocuronium $(\mu \mathrm{g} / \mathrm{kg})$ & - & - & 53,3 & 53,3 \\
\hline
\end{tabular}

Concernant l'halothane, le diazépam, le propofol et la kétamine, les doses moyennes utilisées n'étaient pas significativement différentes entre les MPOE + et MPOE -. Toutefois, comparés aux patients MPO -, les patients $\mathrm{MPO}+$ ont reçu significativement moins d'isoflurane $(\mathrm{t}=2,12, \mathrm{p}=0,0648)$, de kétamine $(\mathrm{t}=1,60, \mathrm{p}=0,1)$, de propofol en bolus $(\mathrm{t}=3,08, \mathrm{p}=0,0025 \%)$, de fentanyl $(\mathrm{t}=2,25, \mathrm{p}=0,02)$ et de pancuronium $(\mathrm{t}=1,47, \mathrm{p}=0,1)$ pour l'entretien de l'anesthésie. Ils ont, par ailleurs, reçu plus d'atropine $(t=3,99$, $\mathrm{p}=0,0002$ ).

Quant au retentissment psychologique de la MPOE, deux patients ont rapporté une anxiété liée à cet événement, un autre a dit ne plus vouloir d'intervention chirurgicale. Les autres patients semblaient plus préoccupés par leur pathologie que par la mémorisation peropératoire. 


\section{Discussion}

Bien qu'il s'est agi d'une étude prospective, nous avons été confrontés à des difficultés concernant le remplissage lacunaire de la feuille d'anesthésie. L’absence de salle de réveil, réputée être idéale pour réaliser la première interview nous a également desservi.

Dans la littérature, la taille des patients à inclure varie de 50 (Hellwagener 2003) à plusieurs milliers (Sebel 2004, Snow 1849). Notre échantillon était 475. Dans notre étude, la majorité des interventions était faite en urgence $(79,1 \%)$ alors que certains auteurs ont étudié les cas électifs (Macario 1999, Ouro-Bang'na 2008,Shi 2006) et d'autres (Snow 1849) les deux. Comme certaines études antérieures (Ranta 1998,Rendshegen 2002) l'ont rapporté, nous avons interrogé les patients une fois alors que d'autres auteurs ont interviewé deux (Hellwagener 2003,Sbel 2004) voir trois fois (Davidson 2005,Sadin 2002,Snow 1849). Un nombre élevé d'interviews permettrait le dépistage (Davidson 2005) malgré les risques de suggestibilité ou de déni au cours d'interrogatoires répétés (Snow 1849). Comme d'autres auteurs (Hellwagener 2003, Shi 2006), nous avons utilisé l'interview de Brice bienque que Enlund et al (Elund 2002, 2006) ont mis en cause sa puissance diagnostique. Malgré cela, cet outil reste celui qui offre le minimum de risque de fausses MPO. Nos patients ont été interrogés en salle d'hospitalisation entre $\mathrm{J} 0$ et $\mathrm{J} 14$, parce qu'il a été démontré que $35 \%$ des cas sont dépistés lors d'une interview retardée (Davidson 2005). Les anesthésistes n'ont pas modifié leurs pratiques au cours de notre étude. Certains auteurs (Rundshagen 2002) n'ont pu garantir une telle discrétion. Lorsque les équipes d'anesthésie sont informées, elles améliorent la qualité des soins, ce qui fausse les résultats (Pollard 2007).

La chirurgie cardiaque, traumatologique, obstétricale, abdominale et ophtalmologique (Tempe 1999) sont réputées à haut risque de MPO. La chirurgie digestive $(75,1 \%)$ prédominait dans notre étude. La plupart des patients dans notre étude (78,1\%) avait un score ASA 3 ou 4. Ranta en 1998 avait inclus des scores ASA plus élevé alors que d'autres auteurs (OuroBang'na maman 2008,Snow 1849) ont inclus des ASA 1 ou 2. Au cours de notre étude, aucune nouvelle drogue n'a été introduite.

Les quantités d'halothane et d'isoflurane délivrées étaient au-dessus de la Concentration Alvéolaire Minimale (MAC) et leur administration était discontinue. Cette discontinuité pourrait être source de réveil et donc de MPO. Ces pratiques anesthésiques ont été décrites dans la littérature (Stait 2008). Au cours de cette étude, le type d'anesthésie le plus pratiqué était appelé Autre type d'Anesthésie (ATA) suivi de l'Anesthésie Balancée. Dans l'étude d'Errando (2008), les techniques d'anesthésie étaient différentes des nôtres. Un monitorage de la profondeur de l'anesthesie n'a pas été utilisé dans notre série. On sait, cependant, que le monitorage physiologique ne 
permet pas toujours de détecter un réveil pendant l'AG même si des auteurs (Sebel 2004) ont trouvé une corrélation. Certains auteurs ont montré que l'utilisation de 1'Index BiSpectral (BIS) réduisait l'incidence de MPO (Ekman 2004, Myles 2004).

Dans notre étude, l'incidence de MPOE était de 1,4\%. Cette incidence est comparable à celle décrite par d'autres auteurs (Snow 1849, Sebel 2004) mais supérieure à celle de Ekman en 2004 et Davidson en 2005.

Le jeune âge constituerait un facteur de risque de MPOE (Brice 1970) en raison d'une résistance accrue aux anesthésiants. Dans notre étude, les patients MPOE+ étaient plus jeunes que les MPOE -. Si certains auteurs n'ont pas mis en évidence un lien entre l'âge et la MPOE (Hellwagner 2003,Shi 2006), d'autres, cependant, (Enlund 2006) ont retrouvé une incidence élevée chez les patients âgés. Dans l'étude de l'ASA Closed Claim (Domino 1999), les femmes avaient plus de MPO que les hommes. Ceci pourrait être dû au fait que les femmes se réveillent plus vite que les hommes suite à l'élimination plus rapide des agents anesthésiques. Dans notre série, il n'y a aucune relation entre sexe et MPOE à l'instar d'autres publications (Hellwagner 2003, Snow 1849). Le groupe des élèves ,étudiants et celui des sans-emploi étaient plus concernés par la MPOE. Le groupe des élèves et étudiants a rapporté le plus grand nombre d'épisodes MPOE ( $\mathrm{p}=$ $0,01)$. Ceci s'explique probablement en partie par le jeune âge et le niveau d'instruction de ce groupe.

$\mathrm{Xu}$ en 2009 a établi un lien entre MPOE et les antécedents d'anesthésie. L'antécédent d'ALR favorisait la survenue de MPOE dans notre série. Ceci pourrait s'expliquer par le fait que les patients s'attendaient à être conscients comme lors d'une ALR et un retour à l'état de conscience pourrait facilement être mémorisé (Veselis 2007). Nous n'avons pas trouvé de lien entre la consommation de substances psychotropes et la survenue de MPO. L'alcool, le tabac, les opiö̈des et les amphétamines ont été incriminés comme des facteurs favorisants (Ghoneim 2000). Selon Domino en 1999, un score ASA 1 ou 2 constitue un facteur de risque, ce qui n'a pas été prouvé dans notre étude. Certains auteurs (Hellwagner 2003, Domino 1999) ont trouvé une association entre MPOE et score ASA élevé qui s'expliquerait par une administration de faibles doses d'anesthésiants en raison de la fragilité des patients agés. Comme Errando en 2008, nous n'avons pas trouvé d'association significative entre le poids et la MPO. Signalons quand-même que l'obésité morbide a été incriminée comme facteur de risque (Veselis 2007) sans pour autant être vérifié (Ghoneim 2009).

Aucune relation entre type de chirurgie et MPOE n'a été trouvée dans notre étude. Ces résultats sont ccomparables à ceux de Errando (Errando 2008) alors que certains auteurs ont établi ce lien (Hellwagner 2003). Six sur 7 MPOE + avaient été opérés en urgence. Selon Errando et al 
(Errando 2008) le contexte d'urgence augmente le risque de MPOE. Certains auteurs ont trouvé une incidence élevée de complications anesthésiques (Aya 1999) et de MPO (Snow 1849) chez les patients opérés la nuit et la fatigue de l'équipe d'anesthésie a été incriminée. Dans notre étude, l'horaire et la durée des interventions n'ont pas influencé l'incidence de MPOE.

Malgré une prémédication aux benzodiazépines, l'incidence de MPOE n'était pas significativement plus basse chez nos patients prémédiqués. Si certaines études (Ho 2001) ont fait la même observation, dans d'autres l'incidence était réduite (Sebel 2004, Snow 1849). Errando (Errando 2008) a trouvé une plus faible incidence de MPO après une prémédication au midazolam qu'aux opioïdes. Le bénéfice d'une prémédication à base d'agents amnésiques dans la prévention de la MPOE a été décrit (Lau 2006).

Une MPO peut survenir au cours de toute AG (Billard 2001) car la stimulation chirurgicale facilite, en soi, l'apprentissage (Deeprose 2004, Mourrise 2007). L'ATIV favoriserait plus la mémorisation qu'une anesthésie balancée (Errando 2008, Miller 1996). Il a été décrit (Sebel 2004, Shi 2006) que l'utilisation de masques laryngés favorisait la MPO. Cependant notre étude, n'a pas établi de relation entre MPOE et mode de contrôle des voies aériennes supérieures. Sachant qu'une grande partie des épisodes de réveil peropératoire a lieu au cours des fortes stimulations, nous pensons que les intubations ont été réalisées sous anesthésie profonde. Les patients MPOE+ ont reçu des doses inférieures de kétamine lorsqu'elle était la principale molécule d'induction, des quantités inférieures d'isoflurane pour l'entretien, des doses inférieures de propofol $(\mathrm{p}=0,002)$ et des doses inférieures de fentanyl $(p=0,02)$. Différents auteurs ont observé un sous dosage des anesthésiants chez les patients MPOE+ (Davidson 2005,Snow 1849). Dans notre étude, les causes probables de MPO étaient l'administration discontinue des drogues et la recherche d'un réveil rapide par l'administration de faibles doses. Chez l'adulte, la MPOE est plus fréquente lorsqu'on utilise des myorelaxants (Davidson 2005). Les curares n'interfèrent pas avec la pharmacocinétique des hypnotiques, n'augmentent pas le risque d'une anesthésie légère mais lorsque celle-ci survient, la curarisation empêche le patient de bouger pour signaler son réveil et retarde ainsi le diagnostic. Un curare a été utilisé chez 4 patients et il n'y avait pas de lien entre MPOE et curarisation ce qui est en adéquation avec l'étude d'Errando (Errando 2008). Sandin et al ont suggéré une utilisation parcimonieuse de myorelaxants qui serait bénéfique pour la prévention de MPO (Sandin 2000).

Aucun patient, dans notre étude, n'a parlé spontanément de MPO avant l'interrogatoire. Il a été rapporté que cette complication n'était pas révélée par les patients par crainte de ne pas être écoutés (Moerman 1993, 
Schwender 1998) ou parce qu'ils ne revoyaient plus leur anesthésiste (Mourisse 2007). Il est décrit que les souvenirs immédiats après un traumatisme engendrent peu ou pas de contenu narratif (Van der kolk 2000). Les patients ont rapporté des souvenirs auditifs et visuels moins traumatisants. L'incidence de perceptions auditives est plus élevée (Snow 1849,Hellwagner 2003) et le risque serait accru avec le propofol (Samuelsson 2007). Des perceptions tactiles et l'anxiété post opératoire surviennent à des fréquences variables (Hellwagner 2003, Mourisse 2007) selon la littérature. Une perception douloureuse rapportée dans la littérature (Hellwagner 2003, Moerman 1993) a été décrite chez une patiente dans notre étude. Plusieurs auteurs ont observé une fréquence élevée de paralysie consciente (Davidson 2005, Mourisse 2007, Hellwagner 2003, Moerman 1993). Des tentatives de « communiquer » (Moerman 1993,Samuelson 2007) à une impression d'asphyxie (Hellwagner 2003) ou la sensation de la présence d'une sonde endotrachéale (Hellwagner 2003,Mourisse 2007) ont été rapportées par les patients. Selon les patients MPOE+ de notre étude, le réveil peropératoire s'est produit en fin d'intervention et a duré quelques secondes. La durée du réveil peropératoire reste relativement inexplorée et difficilement quantifiable. Une étude (Moerman 1993) a néanmoins rapporté une durée moyenne de $7 \mathrm{~min}$.

Selon Aya (Aya 1999), un tiers des anesthésistes dit avoir été confronté à la MPOE mais la majorité de ces anesthésistes l'a considéré comme un évènement mineur. Deux patients ont rapporté une anxiété liée à cet événement et les autres étaient préoccupés par leur pathologie. Le simple souvenir auditif est d'une importance mineure par rapport à la crainte de la chirurgie et de l'hospitalisation (Rundshagen 2002). Dans une étude récente, $21 \%$ des patients ont développé des séquelles à court terme (Sebel 2004) et les séquelles à long terme étaient l'anxiété, la dépression, les cauchemars, les flashbacks et le syndrome de stress post-traumatique (Davidson 2005).

\section{Conclusion}

Malgré le caractère ubiquitaire de ce phénomène, son incidence est faible dans les pays occidentaux qui disposent d'un appareillage sophistiqué permettant de mesurer la profondeur de l'anesthésie. Notre étude a rapporté une incidence de $1,4 \%$. Une anesthésie légère volontaire ou accidentelle, un jeune âge, un niveau d'éducation ou social élevé et des antécédents d'ALR étaient des facteurs associés dans notre étude. La MPO a occasionné un état d'anxiété chez deux de nos patients. Elle peut être prévenue en prémédiquant aux benzodiazépines et en mesurant la profondeur de l'anesthésie. La MPOE avérée nécessite une prise en charge optimale. Une étude multicentrique, regroupant la plupart des spécialités chirurgicales, serait nécessaire pour obtenir l'incidence réelle dans la population générale des opérés au Burkina. 


\section{References:}

1. Aya AGM, Mangin R, Robert C, Ferrer JM, Eledjam JJ.(1999). Increased risk of unintentional dural puncture in night-time obstetric epidural anesthesia. Can J Anesth; 46: 665-9

2. Billard V, Cheikh M .(2001). Réveil et mémorisation peropératoires n'arrivent pas qu'aux autres! Ann Fr Anesth Réanim; 20 : 583-6

3. Brice D, Hetherington RR, Utting JE. (1970). A simple study of awareness and dreaming during anaesthesia. Br J Anaesth; 42: 53542.

4. Davidson AJ, Huang GH, Czarnecki C, Gibson MA, Stewart SA, Jamsen K et al. (2005). Awareness during anesthesia in children : a prospective cohort study. Anesth Analg; 100 : 653-61

5. Deeprose C, Andrade J, Varma S, Edwards N. (2004).Unconscious learning during surgery with propofol anaesthesia. Br J Anaesth; 92: 171-7

6. Domino KB, Posner KL, Caplan RA, Cheney FW.(1999). Awareness during anesthesia: a closed claims analysis. Anesthesiology 90:105361

7. Enlund M, Hassan HG.(2002). Intraoperative awareness: detected by the structured Brice interview? Acta Anaesthesiol Scand; 46:3459.

8. Enlund M.(2006). TIVA, awareness, and the Brice interview. Anesth Analg; 102: 967

9. Ekman A, Lindholm ML, Lennmarken C, Sandin R.(2004). Reduction in the incidence of awareness using BIS monitoring. Acta Anaesthesiol Scand; 48: 20-6

10. Errando CL, Sigl JC, Robles M, Calabuig E, Garcia J, Arocas $F$ et al.(2008). Awareness with recall during general anaesthesia : a prospective observational evaluation of 4,001 patients. $\mathrm{Br} \mathrm{J}$ Anaesth; 101: 178-85

11. Ghoneim MM, Block RI, Haffarnan M, Mathews MJ.(2009). Awareness duringanesthesia : risk factors, causes and sequelae : a review of reported cases in the literature. Anesth Analg; $108: 527-35$

12. Ghoneim MM.(2000). Awareness during anesthesia. Anesthesiology; 92: 597-602

13. Hellwagner K, Holzer A, Gustorff B, Schroegendorfer K, Greher M, Weindlmayr-Goettel M, Saletu B, Lackner F.(2003). Recollection of dreams after short general anaesthesia: Influence on patient anxiety and satisfaction. Eur J Anaesthesiol; 20:282-8

14. Ho AM. (2001). «Awareness » and « recall» during emergence from general anaesthesia. Eur J Anaesthesiol; $18: 623-5$ 
15. Lau K, Matta B, Menon DK, Absalom AR.(2006). Attitudes of anaesthetists to awareness and depth of anaesthesia monitoring in the UK. Eur J Anaesthesiol; 23: 921-30.

16. Macario A, Weinger MN, Truing P, Lee J.(1999). Which clinical anesthesia outcomes are both common and important to avoid? The perspective of a panel of experts of anesthesiologists. Anesth Analg; 88: 1085-91

17. Miller DR, Blew PG, Martineau RJ, Hull KA.(1996). Midazolam and awareness with recall during total intravenous anaesthesia. Can J Anaesth; 43: 946-53

18. Moerman N, Bonke B, Oosting J. (1993). Awareness and recall during general anesthesia. Facts and feelings. Anesthesiology 1993 ; $79: 454-64$

19. Mourisse J, Lerou J, Struys M, Zwarts M, Booij L.(2007). Multilevel approach to anaesthetic effects produced by sevoflurane or propofol in humans: 2. BIS and tetanic stimulus-induced withdrawal reflex. Br J Anaesth; 98: 746-55

20. Myles PS, Leslie K, McNeil J, Forbes A, Chan MT.(2004). Bispectral index monitoring to prevent awareness during anaesthesia: the B-Aware randomised controlled trial. Lancet; 363 : 1757-63

21. Myles PS, Williams DL, Hendrata M, Anderson H, Weeks AM.(2000). Patient satisfaction after anaesthesia and surgery: results of a prospective survey of 10,811 patients. Br J Anaesth; 84: 6-10

22. Ouro-Bang'na maman A.F; Tomta K.; Kangni N. ;Mouzou T. ;Koutou L.; Ahonangbévi S;Chobli M.(2008). Mémorisation peropératoires : enquête auprès de 348 patients au décours d'une anesthésie générale; $15: 186-8$

23. Pollard RJ, Coyle JP, Gilbert RL, Beck JE. (2007). Intraoperative awareness in a regional medical system: a review of 3 years'data. Anesthesiology; $106: 269-74$

24. Ranta SO, Laurila R, saario J, Ali-Melikkilä T, Hynynen M.(1998). Awareness with recall during general anesthesia: incidence and risk factors. Anesth Analg; 86: 1084-9

25. Rundshagen I, Schnabel K,Wegner C, Schulte J.(2002). Incidence of recall, nightmares, and hallucinations during analgosedation in intensive care. Intensive Care Medicine; 28: 38-43

26. Samuelsson P, Brudin L,Sandin RH.(2007).Late psychological symptoms after awareness among consecutively included surgical patients. Anesthesiology; $106: 26-32$ 
27. Sandin RH, Enlund G, Samuelsson P, Lennmarken C.(2000). Awareness during anaesthesia: a prospective case study. Lancet; 355 : 707-11

28. Schwender D, Kunze-Kronawitter H, Dietrich P, Klasing S, Forst H, Madler C.(1998). Conscious awareness during general anaesthesia: patients' perceptions, emotions, cognition and reactions. Br J Anaesth; 80: 133-9

29. Sebel PS, Bowdle T, Ghoneim M, Rampil I, Padilla R, Gan T, Domino K.(2004). The incidence of awareness during anesthesia: A multicenter United States study. Anesth Analg; 99:833-9

30. Shi X, Liu XY, Wang W, Wu XM.(2006). Awareness with recall during general anesthesia: analysis of 2015 cases. Zhonghua Yi Xue Za Zhi. 86:2324-7

31. Snow J.(1849). Use of Chloroform. Lond J Med; 1: 50-5

32. Tempe DK, Siddiquie RA.(1999). Awareness during cardiac surgery. J Cardiothorac Vasc Anesth ; 13: 214-9

33. Stait, M. L.,Leslie, K.,Bailey, R.(2008). Dreaming and recall during sedation for colonoscopy. Anaesthesia and intensive care, 36: 685-90

34. Van der Kolk BA, Osterman JE.(2000). The effects of trauma on memory: implications for awareness under anaesthesia. In: Jordan C, Vaughan DJA, Newton DEF. Memory and Awareness in Anaesthesia IV. London: Imperial College Press: 193-202.

35. Veselis RA. (2007). Memory: a guide for anaesthetists. Best Pract Res Clin Anaesthesiol ; $21: 297-312$

36. Xu L, Wu AS, Yue Y.(2009). The incidence of intra-operative awareness during general anesthesia in China: a multi-center observational study. Acta Anaesthesiol Scand. 53: 873-82. 